\title{
O flúor tópico na redução da cárie dental em adolescentes de Salvador - BA, 1996
}

\section{Topical fluoride in the decline in the prevalence of caries in adolescents from Salvador - BA, 1996}

\author{
Maria Cristina Teixeira CANGUSSU* \\ Maria da Conceição Nascimento COSTA**
}

\begin{abstract}
CANGUSSU, M. C. T.; COSTA, M. da C. N. O flúor tópico na redução da cárie dental em adolescentes de Salvador - BA, 1996. Pesqui Odontol Bras, v. 15, n. 4, p. 348-353, out./dez. 2001.

Os objetivos deste trabalho foram: descrever a experiência de cárie dental em adolescentes de 12 a 20 anos de Salvador - BA e testar a associação entre a severidade da cárie e o uso do flúor tópico na forma de gel ou bochechos. Realizou-se um estudo transversal e análise de regressão logística não condicional, pelo método "backward", com uma amostra de 493 adolescentes escolares do Distrito Sanitário Barra-Rio Vermelho. As variáveis de desenho foram obtidas a partir de questionário semi-estruturado e exame bucal. O CPOD correspondeu a 4,44 (DP 3,71), sendo de 2,72 (DP 2,75 ) aos 12-13 anos. Cento e quarenta e quatro adolescentes participaram de ações de promoção de saúde bucal $(30 \%)$ e a maioria declarou-se pouco apta a manter a própria saúde oral. O flúor tópico não se mostrou como um fator de proteção significativo na redução da cárie dental nesta população $(R P=0,9)$. Houve redução dos índices de cárie dental nos adolescentes do Distrito, embora exista a necessidade de reformulação do modelo cirúrgico-restaurador de atenção à saúde bucal, para maior controle da cárie dental e melhor efetividade dos métodos de promoção e proteção à saúde bucal.
\end{abstract}

UNITERMOS: Cárie dentária; Flúor; Assistência odontológica.

\section{INTRODUÇÃO}

Observa-se, a partir da década de 70, uma significativa redução da prevalência de cárie dental, atribuída à expansão de medidas preventivas com o uso do flúor em países desenvolvidos e, posteriormente, em países em desenvolvimento, confirmada no Brasil nos levantamentos de 1988 e $1996^{2,3}$. Este fenômeno foi acompanhado da polarização da doença, isto é, a concentração da mesma em grupos desfavorecidos social ou economicamente ${ }^{13}$.

Entretanto, o potencial de ação dos métodos tópicos de uso do flúor depende da sua concentração na cavidade oral, da periodicidade do uso do agente protetor e do grau de ataque cariogênico ${ }^{13}$. Dentre os métodos mais empregados, têm-se o uso regular doméstico em baixas concentrações (dentifrícios e bochechos) e o uso esporádico de concentrados de flúor na forma de gel ou verni $z^{11}$.

Os bochechos fluoretados são bastante difundi- dos em programas escolares. Registra-se na literatura redução de $30 \%$ da incidência de cárie dental entre os 6 e 14 anos de idade, sendo necessários pelo menos " 25 bochechos semanais por ano" para obter os efeitos máximos. Porém, os efeitos preventivos desaparecem gradativamente com a interrupção dos programas ${ }^{14}$.

No Brasil, alguns programas escolares tiveram, na década de 80 , o flúor gel como agente preventivo, com sua aplicação através de moldeiras. Entretanto, por questões técnicas, como a não-distribuição do material necessário, questiona-se a sua efetividade $^{15}$. Além disso, principalmente após a década de 80, foram implantados em diversos municípios a "fluorterapia intensiva", em que, além das atividades de educação em saúde, utilizava-se o flúor gel através da escovação e bochechos com flúor em sessões intensivas e de manutenção.

A Faculdade de Odontologia da Universidade Federal da Bahia constitui-se uma unidade docente assistencial, e por isso concentra as suas ações

\footnotetext{
* Mestre em Saúde Comunitária pelo Instituto de Saúde Coletiva, Professora Assistente da Faculdade de Odontologia; **Doutora em Saúde Pública pelo Instituto de Saúde Coletiva, Professora Adjunta do Instituto de Saúde Coletiva - Universidade Federal da Bahia.
} 
CANGUSSU, M. C. T.; COSTA, M. da C. N. O flúor tópico na redução da cárie dental em adolescentes de Salvador - BA, 1996. Pesqui

Odontol Bras, v. 15, n. 4, p. 348-353, out./dez. 2001.

de promoção da saúde bucal e prevenção específica e tratamento reabilitador no Distrito Sanitário Barra-Rio Vermelho, sendo os adolescentes um grupo prioritário. Entretanto, ainda não foram desenvolvidos estudos de efetividade das ações. Nesse sentido, este estudo tem como objetivos descrever a experiência de cárie, fornecer dados sobre a assistência odontológica e determinar a associação entre a exposição aos programas com uso do flúor tópico e a redução da prevalência/severidade da cárie neste grupo.

\section{MATERIAL E MÉTODOS}

Desenvolveu-se um estudo de prevalência ${ }^{12}$ e a população do estudo foi de escolares da quinta série do primeiro grau até a terceira série do segundo grau da rede estadual de ensino, pertencentes a escolas do Distrito Sanitário Barra-Rio Vermelho em Salvador - BA, em 1996, na faixa etária de 12 a 20 anos de idade.

A unidade amostral foi a turma de alunos, utilizando-se no cálculo da amostra um alfa de $5 \%$ e um erro tipo II de $10 \%$, assumindo uma prevalência de $25 \%$ e um efeito de redução de $20 \%$ da doença pelo fator de proteção. O número obtido foi de 14 turmas, totalizando 500 alunos.

Um questionário semi-estruturado, com questões relativas a exposição ao flúor tópico profissional, isto é, ter se submetido ao uso do flúor gel ou verniz em ambiente profissional, ou participação sistemática em programas de bochechos diários ou semanais no ambiente escolar nos últimos dois anos; ter acesso à atenção odontológica curativa, resgatada a partir da pergunta: "completou tratamento restaurador ou de emergência no último ano? Público ou privado?", e uso de medidas de autocuidado (escovação, uso do fio dental e pasta dental fluoretada), dieta (número de refeições com alto consumo de sacarose), além de conhecimentos sobre as causas e métodos preventivos da cárie dental, através de livre resposta foi aplicado. Para o exame bucal, foi proposto o índice CPOD, segundo os critérios propostos pela $\mathrm{OMS}^{9}$, aplicado em ambiente escolar por um único examinador previamente calibrado (concordância de 99,1\%), após a obtenção das autorizações por escrito dos responsáveis (Quadro 1).

Os dados foram digitados no Epi Info 6.04 e a análise foi realizada no SAS 6.12. Procedeu-se à caracterização da população do estudo, à análise univariada e de regressão logística ("backward"), com a razão de prevalência como medida de associação ${ }^{4,5}$, testando-se a significância estatística através do intervalo de confiança (IC) a 95\%, com controle de variáveis de confusão e interação.

A variável dependente foi o $\mathrm{CPO}$, dividido em: sadios, indivíduos com menos de três unidades afetadas, e doentes com 3 dentes ou mais afetados, seguindo a recomendação da meta da OMS. A variável independente principal foi a exposição a pelo menos 3 aplicações tópicas de flúor gel/verniz em ambiente profissional nos últimos 2 anos, através de registro no prontuário escolar ou do serviço de saúde de referência, ou ter participado de modo sistemático de programas diários ou semanais de bochechos fluorados nos últimos 2 anos. As variáveis testadas de confusão e interação foram: idade (menor de 15 anos e 15 anos ou mais), número de escovações/dia, número de refeições com alto consumo de açúcares/dia, exposição a água fluoretada (isto é, ter até 15 anos de idade já que após 1989 houve suspensão deste método no município), consumo de alimentos ricos em flúor (consumo de peixes e mariscos duas vezes por semana ou mais), participação em atividade de educação em saúde no ambiente escolar no último ano e/ou escovação supervisionada, bem como ter se submetido a tratamento odontológico nos últimos 12 meses, incluindo as categorias tratamento emergencial ou tratamento completo.

\section{RESULTADOS}

A população de estudo compreendeu 493 adolescentes, $62 \%$ do sexo feminino, com perda de 7 indivíduos, 6 por evasão escolar e um excluído por ser portador de doença sistêmica. A média de idade foi de 16,4 anos (DP 2,75). O CPOD correspondeu a 4,44 (DP 3,71) (Tabela 1) e, na composição do índice, 40,45\% correspondiam a dentes com cárie, $2,5 \%$ com extração indicada e, $42,45 \%$ de dentes com necessidade de tratamento. Os dentes já obturados representavam $49,77 \%$ e $7,28 \%$ os já extraídos.

Referiu-se uma média de 2,5 ingestões de lanches com alto consumo de sacarose, intercaladas entre as refeições principais. A participação em atividades de promoção de saúde bucal como as ações educativas (palestras, gincanas, minicursos) em saúde bucal e orientações sobre a higiene oral através da escovação supervisionada foram desenvolvidas somente com respectivamente $29,2 \%$ e $35,9 \%$ dos adolescentes do estudo.

Em relação às medidas de proteção específica como o uso de fluoretos, $99,2 \%$ relataram fazer uso diário de pasta dental com flúor e $65,5 \%$ descrevem já terem sido submetidos ao uso tópico de 
CANGUSSU, M. C. T.; COSTA, M. da C. N. O flúor tópico na redução da cárie dental em adolescentes de Salvador - BA, 1996. Pesqui

Odontol Bras, v. 15, n. 4, p. 348-353, out./dez. 2001.

pouco aptos a manter a saúde, por não dominarem as técnicas de autocuidado ou por não terem acesso a escova, dentifrício ou fio dental por restrições financeiras.

Considerando-se a exposição ao flúor tópico como um possivel fator para os menores índices de cárie dental, verificou-se na análise descritiva que os grupos dos expostos e não-expostos ao fator se diferenciavam principalmente em relação à idade. Os que relataram o uso do flúor tópico eram os mais jovens e os que consumiam uma dieta mais rica em açúcares (Tabela 2).

Existiu uma associação negativa entre cárie dental e a exposição ao flúor tópico na análise univariada, não estatisticamente significante. Já na análise tabular estratificada, duas variáveis pareceram interferir nesta associação como modificadores de efeito - a participação em atividades de escovação supervisionada e o alto consumo de carboidratos. Observou-se um maior efeito protetor do flúor tópico nos indivíduos que nunca participaram de escovação supervisionada e naqueles que relatam alto consumo de açúcar (Tabela 3).

Na regressão logística, nenhum termo de interação foi estatisticamente significante, com um valor de $\mathrm{p}$ obtido da estatítisca do qui-quadrado de 0,455 a partir dos valores observados da razão de máxima verossimilhança. Ao se avaliar a interferência de variáveis relevantes à associação, permaneceram como co-variáveis a idade e a assistência odontológica (tratamento completo). A primeira, como um fator de risco importante para a cárie dental nas idades mais jovens e o tratamento odontológico como fator protetor. Observou-se um pequeno efeito do flúor profissional na redução da cárie dental, não estatisticamente significante (Tabela 4).

TABELA 1 - Índice CPOD (média e desvio-padrão) e percentual de indivíduos livres de cárie dental por idade em adolescentes escolares de Salvador - BA, 1996.

\begin{tabular}{c|c|c|c}
\hline \hline $\begin{array}{c}\text { Idade } \\
\text { (anos) }\end{array}$ & $\mathrm{n}$ & $\begin{array}{c}\text { CPOD e } \\
\text { desvio-padrão }\end{array}$ & $\begin{array}{c}\text { \% de indivíduos } \\
\text { livres de cárie }\end{array}$ \\
\hline $12-13$ & 81 & $2,7(2,8)$ & 28,2 \\
\hline 14 & 83 & $3,3(3,5)$ & 24,1 \\
\hline 15 & 88 & $3,7(2,5)$ & 18,8 \\
\hline 16 & 74 & $4,3(3,2)$ & 12,2 \\
\hline 17 & 60 & $4,5(3,4)$ & 13,2 \\
\hline 18 & 55 & $5,1(3,6)$ & 13,8 \\
\hline $19-20$ & 52 & $6,9(4,3)$ & 7,6 \\
\hline Total & 493 & $4,4(3,7)$ & 16,6 \\
\hline \hline
\end{tabular}

\section{DISCUSSÃO}

Confirmou-se neste estudo a redução significativa da cárie dental em adolescentes no Distrito Sanitário Barra-Rio Vermelho em Salvador - BA, com um CPOD de 2,72 (DP 2,75) aos 12 anos de idade, valor semelhante ao relatado pelo Ministério da Saúde no ano de 1996 para o Brasil ${ }^{3}$. Entretanto, a média foi maior do que a encontrada neste mesmo levantamento para o município de Salvador, o que pode ser decorrente de diferenças metodológicas entre os estudos, especialmente a reconhecida subestimação da cárie dental decorrente da incorreta interpretação dos componentes presença de cavitação/mancha branca no período.

A meta proposta pela OMS para o ano 2000 aos 12 anos de idade, de menos de três dentes acometidos pela cárie dentária, já foi cumprida por este grupo. Contudo, um aspecto de extrema relevância é que, aos 18 anos, segundo esta própria orga-

TABELA 2 - Distribuição percentual dos fatores de risco e de proteção segundo a exposição ao flúor tópico em 493 adolescentes escolares de Salvador - BA, 1996.

\begin{tabular}{|c|c|c|c|}
\hline Fatores estudados & $\begin{array}{c}\text { Não } \\
\mathrm{n}=169\end{array}$ & $\begin{array}{c}\text { Sim } \\
\mathrm{n}=324\end{array}$ & $\begin{array}{c}\text { Valor } \\
\text { de p }\end{array}$ \\
\hline \multicolumn{4}{|l|}{ Idade } \\
\hline $12-16$ anos & 37,87 & 42,72 & \multirow{2}{*}{0,05} \\
\hline Acima de 17 & 62,13 & 57,28 & \\
\hline \multicolumn{4}{|l|}{ Assistência odontológica } \\
\hline Nunca & 84,61 & 57,28 & 0,28 \\
\hline Urgência e TC & 15,39 & 42,72 & \\
\hline
\end{tabular}

Atividade de educação em saúde

\begin{tabular}{c|l|l|l}
\hline Sim & 13,00 & 37,77 & \multirow{2}{*}{0,26} \\
\cline { 1 - 3 } Não & 87,00 & 62,23 & \\
\hline
\end{tabular}

Escovação supervisionada

\begin{tabular}{c|l|l|l}
\hline Sim & 13,60 & 47,68 & \multirow{2}{*}{0,34} \\
\cline { 1 - 2 } Não & 86,40 & 52,32 & \\
\hline
\end{tabular}

Alto consumo de açúcares

\begin{tabular}{c|l|l|l}
\hline Sim & 42,50 & 47,68 & \multirow{2}{*}{0,09} \\
\cline { 1 - 3 } Não & 57,50 & 52,32 & \\
\hline
\end{tabular}

Exposição ao flúor na água

\begin{tabular}{c|c|c|c}
\hline Sim & 95,00 & 94,12 & \multirow{2}{*}{0,21} \\
\cline { 1 - 3 } Não & 5,00 & 5,88 & \\
\hline \hline
\end{tabular}

$\mathrm{TC}=$ tratamento completo. 
CANGUSSU, M. C. T.; COSTA, M. da C. N. O flúor tópico na redução da cárie dental em adolescentes de Salvador - BA, 1996. Pesqui

Odontol Bras, v. 15, n. 4, p. 348-353, out./dez. 2001.

TABELA 3 - Análise da associação bruta e estratificada entre cárie dental e exposição ao flúor tópico em adolescentes escolares de Salvador - BA, 1996.

\begin{tabular}{l|c|c|c|c}
\hline \hline \multirow{2}{*}{ Fatores estudados } & $\mathrm{n}$ & \multirow{2}{*}{$\mathrm{RP}$} & \multicolumn{2}{|c}{$\begin{array}{c}\text { Intervalo de } \\
\text { confiança - 95\% }\end{array}$} \\
\cline { 4 - 5 } & & & Mínimo & Máximo \\
\hline Associação crua & 493 & 0,94 & 0,79 & 1,12 \\
\hline Idade \\
\hline $12-16$ anos & 326 & 0,92 & 0,78 & 1,08 \\
\hline Acima de 17 & 167 & 0,94 & 0,80 & 1,12 \\
\hline
\end{tabular}

Assistência odontológica

\begin{tabular}{c|c|c|c|c}
\hline Nunca + urgência & 328 & 0,93 & 0,75 & 1,14 \\
\hline TC & 165 & 1,08 & 0,77 & 1,50 \\
\hline
\end{tabular}

Atividade de educação em saúde

\begin{tabular}{c|c|c|c|c}
\hline Sim & 144 & 1,06 & 0,69 & 1,60 \\
\hline Não & 349 & 0,89 & 0,74 & 1,08 \\
\hline
\end{tabular}

Escovação supervisionada

\begin{tabular}{c|c|c|c|c}
\hline Sim & 178 & 1,29 & 0,92 & 1,80 \\
\hline Não & 315 & 0,83 & 0,68 & 1,02 \\
\hline
\end{tabular}

Alto consumo de açúcares

\begin{tabular}{c|c|c|c|c}
\hline Sim & 301 & 0,84 & 0,66 & 1,07 \\
\hline Não & 192 & 1,09 & 0,84 & 1,42 \\
\hline
\end{tabular}

Exposição ao flúor na água

\begin{tabular}{c|r|r|r|r}
\hline Sim & 456 & 1,12 & 0,92 & 1,35 \\
\hline Não & 37 & 0,95 & 0,74 & 1,24 \\
\hline \hline
\end{tabular}

$\mathrm{RP}=$ razão de prevalência; $\mathrm{TC}=$ tratamento completo.

nização, os jovens deveriam ter subsídios suficientes para o controle das doenças bucais. Neste trabalho, observou-se que ou por limitações socioeconômicas ou por não terem sido adequadamente capacitados pelas técnicas de educação em saúde bucal, a maior parte dos adolescentes (79\%) não se sente apto a prevenir futuros problemas bucais.

O acesso à assistência odontológica de 33,5\% é maior do que proporções citadas em outros levantamentos nacionais, que variam entre $4 \%$ e $25 \%$, demonstrando uma expansão da assistência odontológica, nos setores público e privado conveniado, no município de Salvador. Como conseqüência, quando se analisa a composição do índice CPOD, predominam os dentes restaurados e extraídos em relação àqueles com necessidade de tratamento (cariados e com extração indicada).

O restante da população, 66,5\% do grupo, não
TABELA 4 - Associação obtida através da regressão logística entre exposição ao flúor profissional e cárie dental em 493 adolescentes escolares de Salvador - BA, 1996.

\begin{tabular}{|c|c|c|c|}
\hline \multirow[t]{2}{*}{ Co-variáveis } & \multirow[t]{2}{*}{$\mathrm{RP}$} & \multicolumn{2}{|c|}{$\begin{array}{c}\text { Intervalo de } \\
\text { confiança - 95\% }\end{array}$} \\
\hline & & Mínimo & Máximo \\
\hline Associação principal & 0,9 & 0,6 & 1,3 \\
\hline Idade & 3,1 & 2,1 & 4,5 \\
\hline Tratamento odontológico & 0,7 & 0,5 & 1,1 \\
\hline
\end{tabular}

tem acesso a tratamento reabilitador ou preventivo, recorrendo ao atendimento de urgência em casos de dor. Este tipo de atenção reproduz um modelo de prática mutiladora, vigente no país desde o início do século, de alto custo e baixo impacto epidemiológico ${ }^{8}$.

A fluoretação das águas de abastecimento público, mesmo com a suspensão do método pela empresa de abastecimento pública em 1989, beneficiou uma parcela significativa dos adolescentes no periodo das unidades dentárias permanentes ${ }^{1}$. O flúor sob a forma de gel, usado em aplicações tópicas profissionais e o flúor em solução, utilizado em programas de bochechos escolares, embora muito utilizados por este grupo não se mostraram programas de grande efetividade na redução da cárie dentária, apesar de autores como PINTO $^{13}$ e STERRIT et al. ${ }^{14}$ relatarem reduções de cerca de $30 \%$ das lesões em estudos longitudinais com a regularidade de pelo menos uma aplicação semestral e 25 sessões semanais, respectivamente.

O efeito protetor obtido, de $10 \%$, aproxima-se dos resultados descritos por MOREIRA ${ }^{7}$ e vários fatores podem ter contribuído para a menor efetividade destes métodos: as interrupções freqüentes no calendário escolar e não cumprimento da periodicidade adequada ao programa de bochechos; uso de quantidades insuficientes de flúor ou dificuldades no desenvolvimento da técnica adequada; dificuldades em garantir no domicílio a continuidade das ações de promoção da saúde através do autocuidado e a predominância nos serviços municipais de saúde de um modelo assistencial cirúrgico-restaurador, que pouco valoriza ou enfatiza as ações coletivas em saúde bucal ou mesmo preventivas no âmbito individual.

$\mathrm{O}$ consumo de alimentos ricos em sacarose e carboidratos, que atingiu uma média de 2,5 refeições intercaladas às principais, foi um resultado próximo ao descrito por MAJEM et $a .^{5}$ na Espa- 
CANGUSSU, M. C. T.; COSTA, M. da C. N. O flúor tópico na redução da cárie dental em adolescentes de Salvador - BA, 1996. Pesqui

Odontol Bras, v. 15, n. 4, p. 348-353, out./dez. 2001.

nha. Os autores ${ }^{5,13}$ atribuem este comportamento ao baixo custo deste grupo de alimentos, bem como a sua grande valorização na cultura e dieta ocidentais. Nos adolescentes que relataram três ou mais refeições com alto consumo de sacarose ou carboidratos, foi observado um maior efeito protetor do flúor (30\%), reforçando a importância destes métodos nas populações de alto risco à cárie den$\operatorname{tal}^{13}$.

\section{CONCLUSÕES}

O uso do flúor tópico profissional na forma de gel ou bochechos não foi relevante para detectar diferenças na prevalência de cárie dentária neste grupo.

Houve significativa redução dos índices de cárie dental, tendo sido atingida a meta proposta pela OMS para o ano 2000.

Considerou-se pouco satisfatória a indicação de que, aos 18 anos de idade, os adolescentes considerem-se pouco aptos a manter sua própria saúde bucal, por limitações econômicas e de fragilidades nas atividades de educação em saúde bucal.

A cobertura da assistência odontológica foi maior do que a detectada em outros estudos, sendo uma variável relevante em relação à prevalência de cárie dental, assim como a cobertura de métodos preventivos e de promoção da saúde bucal.

É necessário repensar as práticas docente-assistenciais desenvolvidas no Distrito de forma a desenvolver ações mais efetivas no controle da cárie dental.

CANGUSSU, M. C. T.; COSTA, M. da C. N. Topical fluoride in the decline in the prevalence of caries in adolescents from Salvador - BA, 1996. Pesqui Odontol Bras, v. 15, n. 4, p. 348-353, out./dez. 2001.

The aim of this paper was to investigate the experience of dental caries in a population of young adults from Salvador $\mathrm{BA}$, and to associate the prevalence of caries with the utilization of topical fluoride. A cross-sectional study was carried out with a sample of 493 persons aged 12-20 years, from Salvador - BA. Data were obtained from interviews and clinical examinations and they were analyzed through backward logistic regression. The DMFT was 4.44 (SD 3.71), and, for subjects aging 12-13 years, it was 2.72 (SD 2.75). A hundred and forty adolescents reported that they had participated in oral health programs $(30 \%)$, and the majority felt that they were unable to maintain their own oral health. Topical fluoride was not a significant factor in the decrease of the prevalence of caries among these young adults $(\mathrm{RP}=0.9)$. There was a decline in dental caries in this group. However, it is necessary to review the surgical-restorative model of dental practice in order to achieve better results in the promotion of oral health.

UNITERMS: Dental caries; Fluorine; Dental care.

\section{REFERÊNCIAS BIBLIOGRÁFICAS}

1. BAHIA. SESAB. Plano Estadual de Saúde 1996-1999. Salvador, 1996, p. 86-93.

2. BRASIL. Ministério da Saúde. Levantamento Epidemiológico em Sáude Bucal: Brasil, zona urbana, 1986. 1988, 137 p.

3. CFO. Jornal informativo. Brasília, março 1997. 8 p.

4. KLEINBAUN, D. G.; KUPPER, L. L.; MORGENSTERN, H. Epidemiologic research: principles and quantitative methods. Van Nostrnd Reinhold, 1982, 529 p.

5. KLEINBAUN, D. G. Logistic Regression - a self learning text. New York : Springer Verlag, 1994. 282 p.

6. MAJEM, L. S.; GARCIA, C. R.; RAMOS, J. M. Dietary habits and dental caries in a population of Spanish schoolchildren with low levels of caries experience. Caries Res, v. 27, n. 6, p. 488-494, 1993.

7. MOREIRA, E. J. G.; NARESSI, W. G.; VANTINE, A. S. Bochechos fluorados. Rev Assoc Paul Cir Dent, v. 41, n. 1, p. 18-23, 1987.
8. NARVAI, P. C. Odontologia e Saúde Bucal Coletiva. São Paulo : HUCITEC/ABRASCO, 1994. 113 p.

9. ORGANIZAÇÃO MUNDIAL DA SAÚDE. Oral health surveys: basic methods. 3. ed. Geneva, 1987, p. 1-53.

10. ORGANIZAÇÃO MUNDIAL DA SAÚDE. DMFT levels at 12 years. Geneva, 1995. $17 \mathrm{p}$.

11. O'MULlane, D. Can prevention eliminate caries? Adv Dent Res, v. 9, n. 2, p. 106-109, July 1995.

12. PEREIRA, M. G. Epidemiologia: teoria e prática. Rio de Janeiro : Guanabara Koogan, 1995, 583 p.

13. PINTO, V. G. Saúde Bucal: odontologia social e preventiva. 3. ed. São Paulo : Santos, 1992. 415 p.

14. STERRIT, G. R.; FREW, R. A.; ROZIER, R. G. Evaluation of Guamanian dental caries preventive programs. J Public Health Dent, v. 54, n. 3, p. 153-159, 1994.

15. ZANETTI, C. H. G.; LIMA, M. A. V.; RAMOS, L.; COSTA, M. A. B. T. Em busca de um paradigma de programação local em saúde bucal mais resolutivo no SUS. Divulgação em Saúde para Debate, n. 13, p. 18-35, jul. 1996. 